\title{
Clinical Guideline Highlights for the Hospitalist: Clostridium difficile Infections in Children
}

\author{
Amanda Rogers, MD*, Abdul ElKadri, MD
}

Department of Pediatrics, Medical College of Wisconsin, Milwaukee, Wisconsin.

GUIDELINE TITLE: Clinical Practice Guidelines for Clostridium difficile Infection in Adults and Children: 2017 Update by the Infectious Diseases Society of America (IDSA) and Society for Healthcare Epidemiology of America (SHEA).

RELEASE DATE: February 15, 2018

PRIOR VERSIONS:

- Cohen SH, Gerding DN, Johnson S, et al; Society for Healthcare Epidemiology of America; Infectious Diseases Society of America. Clinical practice guidelines for Clostridium difficile infection in adults: 2010 update by the Society for Healthcare Epidemiology of America (SHEA) and the Infectious Diseases Society of America (IDSA). Infect Control Hosp Epidemiol. 2010; 31:431-55.

- Gerding DN, Johnson S, Peterson LR, et al. Clostridium difficile-associated diarrhea and colitis. Infect Control Hosp Epidemiol 1995;16:459-477.

DEVELOPER: IDSA and SHEA.

FUNDING SOURCE: Support for this guideline was provided by the IDSA and SHEA.

TARGET POPULATION: Children and adults with Clostridium difficile infections.

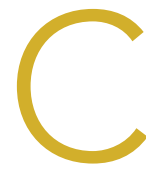

lostridium difficile (name changed to Clostridioides difficile [CDI]) are a major public health problem, with 500,000 infections annually in the United States,

15,000-30,000 associated deaths, and acute care costs exceeding $\$ 4.8$ billion. The recent clinical practice guideline for CDI provides recommendations about the epidemiology, diagnosis, treatment, prevention, and environmental management. A total of 52 recommendations are included, and we will review 11 with pertinence to pediatrics in this highlight.

\section{KEY RECOMMENDATIONS FOR THE HOSPITALIST}

Recommendation 1. In infants $\leq 12$ months of age, testing for $\mathrm{CDI}$ should never be routinely recommended because of the high prevalence of asymptomatic carriage of toxigenic $\mathrm{C}$. difficile in infants (strong recommendation, moderate quality of evidence).

Recommendation 2. In children 1-2 years of age, testing should not be routinely performed unless other causes have been excluded (weak recommendation, low quality of evidence).

Recommendation 3 . In children $\geq 2$ years of age, testing is recommended for patients with prolonged or worsening di-

*Corresponding Author: Amanda Rogers, MD; E-mail: arogers@mcw.edu; Telephone: 414-337-7050.

Published online first October 23, 2019

Received: May 30, 2019; Revised: August 11, 2019; Accepted: August 19, 2019

(c) 2020 Society of Hospital Medicine DOI 10.12788/jhm.3312 arrhea and risk factors (eg, underlying inflammatory bowel disease) or immunocompromising conditions) or relevant exposures (eg, contact with the healthcare system or recent antibiotics) (weak recommendation, moderate quality of evidence).

The rate of $C$. difficile colonization among asymptomatic infants can exceed $40 \%$. This rate declines over the first year but remains $15 \%$ at 12 months of age. ${ }^{1}$ Therefore, the guideline recommends against routinely testing infants $\leq 12$ months of age as a positive test probably reflects colonization rather than disease. Testing in infants is recommended only when other causes have been excluded and a concern for pseudomembranous colitis, toxic megacolon, or clinically significant diarrhea exists.

The rate of asymptomatic colonization remains elevated in the second year of life. By 2-3 years, the rate is 1\%-3\% which is similar to that in healthy adults. However, the role of $C$. difficile in community-onset diarrhea in otherwise healthy children is controversial. In a study of 100 hospitalized children aged $<2$ years with CDI and diarrhea, all had resolution of diarrhea regardless of whether therapy was administered. ${ }^{2}$ Another study found an alternative pathogen in $>50 \%$ of hospitalized children with CDI. ${ }^{3}$ Therefore, the guideline recommends against testing in children aged 1-2 years unless other causes have been excluded and in children aged $>2$ years only when they have prolonged or worsening diarrhea along with risk factors or exposures.

Recommendation 4. In institutions without specific required criteria for stool submissions, use a stool toxin test as part of a 
multistep algorithm (ie, glutamate dehydrogenase [GDH] plus toxin, GDH plus toxin arbitrated by nucleic-acid amplification tests [NAAT], or NAAT plus toxin) rather than a NAAT alone (weak recommendation, low quality of evidence).

Recommendation 5. In institutions with specific required criteria for stool submissions, use a NAAT alone or a multistep algorithm for testing (ie, GDH plus toxin, GDH plus toxin arbitrated by NAAT, or NAAT plus toxin) rather than a toxin test alone (weak recommendation, low quality of evidence).

There are a variety of testing approaches for $\mathrm{CDI}$ and recommendations vary based on local practice. If laboratories accept all stools, a more specific approach is recommended, including a toxin test as part of a multistep algorithm to limit false positives. If laboratories first screen for symptoms and antibiotic exposure before accepting stool samples, a more sensitive approach is recommended including NAAT alone or a multistep algorithm rather than toxin alone.

\section{Infection Prevention and Control}

Recommendation 6. There is insufficient evidence for discontinuation of PPIs (proton pump inhibitors) as a measure for preventing $\mathrm{CDI}$ (no recommendation).

The guideline acknowledges data suggesting an association between PPI use and CDI, but not a causal relationship. Due to the lack of high-quality evidence, it does not recommend stopping PPIs to prevent CDI.

Recommendation 7. There are insufficient data to recommend probiotics for primary prevention of CDI outside of clinical trials (no recommendation).

The guideline notes that although several meta-analyses indicate that probiotics may prevent CDI; however there were limitations, including a high incidence of CDI in placebo arms and differences in probiotic formulations and duration of use, leading to insufficient data to recommend probiotic use to prevent CDI.

\section{Treatment}

Recommendation 8. Either per os (PO) metronidazole or PO vancomycin is recommended for an initial episode or first recurrence of nonsevere pediatric CDI (weak recommendation, low quality of evidence).

Data assessing the optimal treatment for nonsevere pediatric $\mathrm{CDI}$ are limited. Emerging data support the use of vancomycin, ${ }^{4}$ which is now recommended for initial episodes of CDI in adults. However, there are insufficient data to recommend vancomycin over metronidazole for nonsevere pediatric CDI; therefore, either option is recommended.

Recommendation 9. For children with an initial episode of severe CDI, oral vancomycin with or without IV metronidazole is recommended over metronidazole alone (strong recommendation, moderate quality of evidence).

Recommendation 10. For children with a second or greater episode of recurrent $\mathrm{CDI}$, oral vancomycin is recommended over metronidazole (weak recommendation, low quality of evidence).

There is no well-designed trial comparing metronidazole and vancomycin for severe or recurrent pediatric CDI. For children previously treated with metronidazole, vancomycin is recommended based on adult literature. ${ }^{4}$ For children previously treated with metronidazole and vancomycin, an extended course of tapered or pulse regimen vancomycin or vancomycin followed by rifaximin is recommended.

Recommendations must weigh potential harms. Metronidazole has been associated with neuropathies, ${ }^{5}$ cramping, and nausea. PO vancomycin has poor enteral absorption, minimizing systemic effects. Both vancomycin and metronidazole may promote carriage of resistant enterococci.

Recommendation 11. Fecal microbiota transplantation (FMT) should be considered for pediatric patients with multiple recurrences of CDI following standard treatments (weak recommendation, very low quality of evidence).

There are no robust data examining the effectiveness of pediatric FMT. Recommendations are guided by adult studies. Limited evidence suggests that FMT can be effective in children with multiple recurrent CDI. ${ }^{6}$ Concerns include procedure-related risks, transmission of resistant organisms and blood-borne pathogens, and induced metabolic or immunologic disorders.

\section{CRITIQUE}

\section{Methods in Preparing a Guideline}

The strength of a guideline includes representation from a diverse panel, including the Infectious Diseases Society of America (IDSA), the Society for Healthcare Epidemiology of America, the American Society of Health-Systems Pharmacists, the Society of Infectious Diseases Pharmacists, and the Pediatric Infectious Diseases Society.

The panel utilized the Grading of Recommendations Assessment, Development, and Evaluation system to weigh the strength and quality of evidence.

From a pediatric perspective, the current guideline added pediatric-specific recommendations based on a comprehensive review of the literature from 1977 to 2016. The strength of these recommendations is somewhat limited by the lack of well-designed pediatric studies. An additional limitation is that treatment recommendations are based on illness severity, although the definitions used to classify severity are not pediatric-specific and are based on unvalidated expert opinion.

\section{Sources of Potential Conflicts or Interest or Bias}

The panel complied with the IDSA policy on conflicts of interest and disclosed any interest that might be construed as a conflict, regardless of relevancy. These were evaluated by the IDSA Standards and Practice Guidelines Committee.

\section{Generalizability}

Guideline generalizability may be impacted by testing availabilities within a particular setting. Cost factors and local formularies may also limit treatment options within a given setting.

\section{Areas in Need of Future Study}

Research gaps exist regarding at what age $C$. difficile is pathogenic given the prevalence of asymptomatic carriage. Future 
studies can also focus on a newly available molecular polymerase chain reaction test platform that detects $C$. difficile. ${ }^{7}$

There is limited pediatric evidence to recommend metronidazole versus vancomycin in children, particularly in nonsevere cases. There is also an opportunity to further explore alternative therapies, including fidaxomicin (not currently approved for children) and bezlotoxumab, a new agent approved as adult adjunctive therapy. ${ }^{8}$

Disclosures: Dr. Rogers and Dr. ElKadri have nothing to disclose.

\section{References}

1. Donta ST, Myers MG. Clostridium difficile toxin in asymptomatic neonates. J Pediatr. 1982;100(3):431-434. https://doi.org/10.1016/s0022-3476(82)80454-x.

2. González-Del Vecchio $M$, Álvarez-Uria A, Marin $M$, et al. Clinical significance of Clostridium difficile in children less than 2 years old: a case-con- trol study. Pediatr Infect Dis J. 2016;35(3):281-285. https://doi.org/10.1097/ INF.0000000000001008.

3. Valentini D, Vittucci AC, Grandin A, et al. Coinfection in acute gastroenteritis predicts a more severe clinical course in children. Eur J Clin Microbiol Infect Dis. 2013;32(7):909-915. https://doi.org/10.1007/s10096-013-1825-9.

4. Johnson S, Louie TJ, Gerding DN, et al. Vancomycin, metronidazole, or tolevamer for Clostridium difficile infection: results from two multinational, randomized, controlled trials. Clin Infect Dis. 2014;59(3):345-354. https://doi. org/10.1093/cid/ciu313

5. Yamamoto T, Abe K, Anjiki H, Ishii T, Kuyama Y. Metronidazole-induced neurotoxicity developed in liver cirrhosis. J Clin Med Res. 2012;4(4):295-298. https://doi.org/10.4021/jocmr893w.

6. Russell G, Kaplan J, Ferraro M, Michelow IC. Fecal bacteriotherapy for relapsing Clostridium difficile infection in a child: a proposed treatment protocol. Pediatrics. 2010;126(1):e239-e242. https://doi.org/10.1542/peds.20093363.

7. Zhang H, Morrison S, Tang YW. Multiplex polymerase chain reaction tests for detection of pathogens associated with gastroenteritis. Clin Lab Med. 2015;35(2):461-486. https://doi.org/10.1016/j.cll.2015.02.006.

8. Wilcox MH, Gerding DN, Poxton IR, et al. Bezlotoxumab for prevention of recurrent Clostridium difficile infection. N Engl J Med. 2017;376(4):305-317. https://doi.org/10.1056/NEJMoa1602615. 